\title{
Dynamic Walking on Randomly-Varying Discrete Terrain with One-step Preview
}

\author{
Quan Nguyen, Ayush Agrawal, Xingye Da, William C. Martin, \\ Hartmut Geyer, Jessy W. Grizzle, and Koushil Sreenath.
}

\begin{abstract}
An inspiration for developing a bipedal walking system is the ability to navigate rough terrain with discrete footholds like stepping stones. In this paper, we present a novel methodology to overcome the problem of dynamic walking over stepping stones with significant random changes to step length and step height at each step. Using a 2-step gait optimization, we not only consider the desired location of the next footstep but also the current configuration of the robot, thereby resolving the problem of step transition when we switch between different walking gaits. We then use gait interpolation to generate the desired walking gait in real-time. We demonstrate the method on a planar dynamical walking model of ATRIAS, an underactuated bipedal robot walking over a randomly generated stepping stones with step length and step height changing in the range of [30:80] $(\mathrm{cm})$ and [30:30] $(\mathrm{cm})$ respectively. Experimental validation on the real robot was also successful for the problem of dynamic walking on stepping stones with step lengths varied within $[23: 78](\mathrm{cm})$ and average walking speed of $0.6(\mathrm{~m} / \mathrm{s})$.
\end{abstract}

\section{INTRODUCTION}

The ability of traversing rough terrain with discrete footholds such as "stepping stones" is a primary advantage of robotic systems with legged locomotion modalities. While current methods rely on simplistic methods, both at the level of models of bipedal robots (e.g., linear inverted pendulum) and control (e.g., ZMP) to achieve the desired foot placements via static walking, we will propose here a formal framework to achieve dynamic walking over a randomly placed, widely varying set of stepping stones with significant changes on step length and step height at each step.

Footstep placement control for fully actuated legged robots initially relied on quasi-static walking and resulted in slow walking speeds [12],[13],[4]. Impressive results in footstep planning and placements in obstacle filled environments with vision-based sensing have been carried out in [14],[3]. The DARPA Robotics Challenge inspired several new methods,

The work by Q. Nguyen, A. Agrawal, and K. Sreenath is supported by NSF grant IIS-1526515. The work by X. Da and J. W. Grizzle is supported by NSF grants IIS-1525006, CNS-1239037. The work by W. C. Martin and H. Geyer is supported by NSF grant CNS-1239143.

Q. Nguyen, A. Agrawal, and K. Sreenath are with the Dept. of Mechanical Engineering, Carnegie Mellon University, Pittsburgh, PA 15213, email: \{qtn, ayushagrawal, koushils\}ecmu.edu.

$\mathrm{X}$. Da is with the Dept. of Mechanical Engineering, University of Michigan, Ann Arbor, MI 48109, email: xda@umich.edu.

W. C. Martin and H. Geyer are with the Robotics Institute, Carnegie Mellon University, Pittsburgh, PA 15213, email: \{wmartin, hgeyer\} @ cmu.edu.

J. W. Grizzle is with the Dept. of Electrical Engineering and Computer Science, University of Michigan, Ann Arbor, MI 48109, email: grizzle@umich.edu.

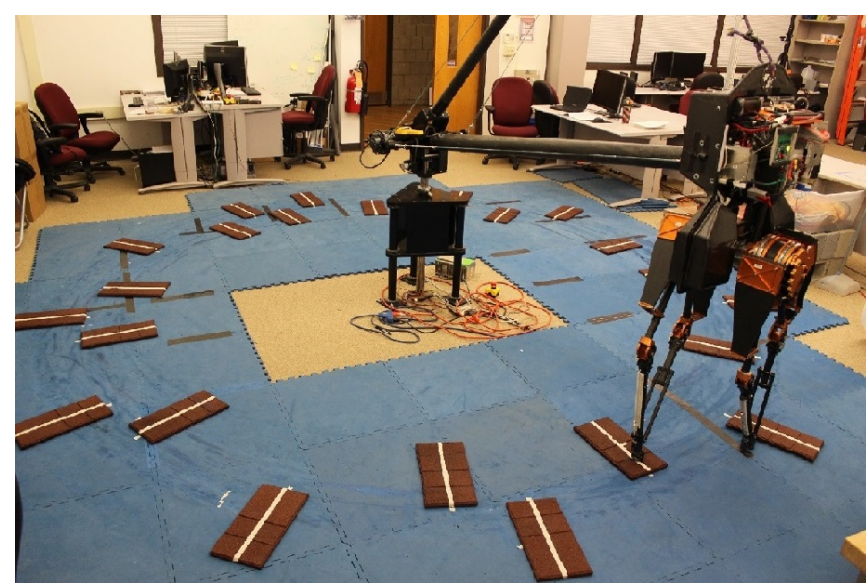

(a)

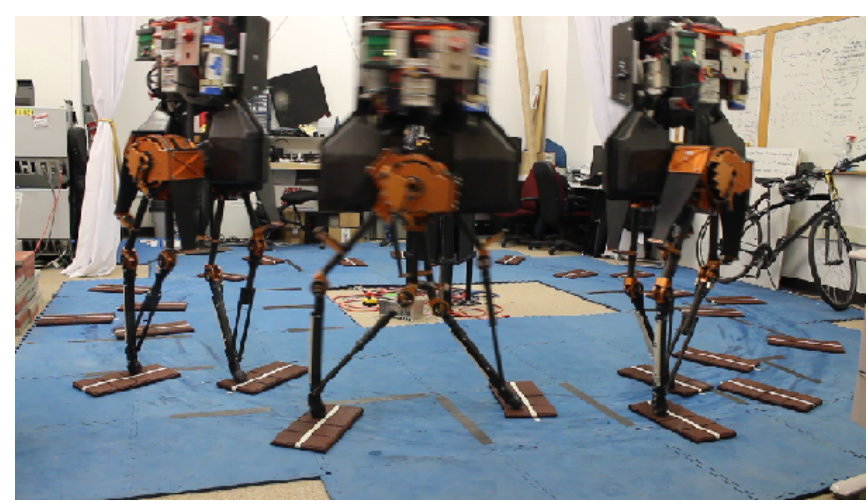

(b)

Fig. 1: ATRIAS walking over stepping stones. Experimental video: https://youtu.be/JKkPWHm6H7k. Simulation video: https://youtu.be/Pxhb4_ojiC8

some based on mixed-integer quadratic programs [7]. However, as mentioned in [8, Chap. 4], mixed-integer-based footstep planning does not offer dynamic feasibility even on a simplified model. These methods are therefore not applicable for dynamic walking with faster walking gaits. On the other hand, the approach developed in [22] allows aperiodic gaits with varying step lengths designed on a complete dynamical model, but requires the a priori design of controllers that realize precise transitions between each pair of elements of the gait library, resulting in exponential (factorial) growth in the number of pre-designed controllers.

In this paper, instead of relying on kinematics of quasistatic motion planning of simplified dynamical models such as the linear inverted pendulum with massless legs [9],[18], we present a novel control strategy based on the full non- 


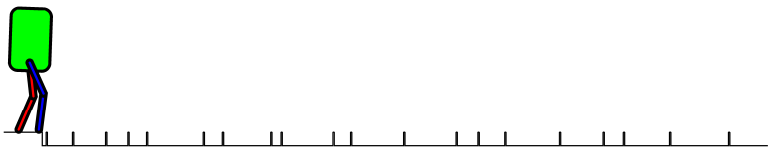

(a) Changing Step Length

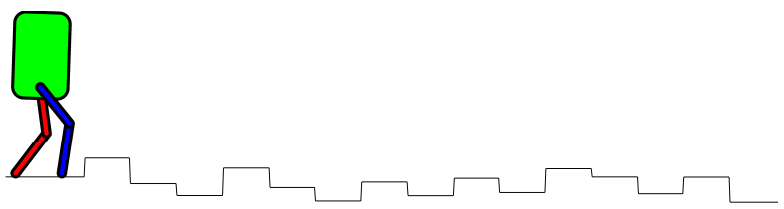

(b) Changing Step Height

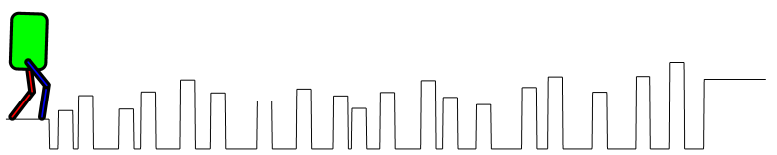

(c) Changing both Step Length and Step Height

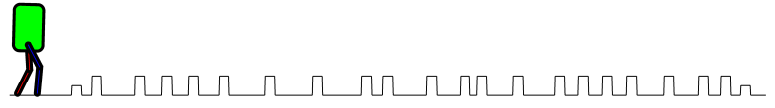

(d) Planar Version of the W-Prize Terrain

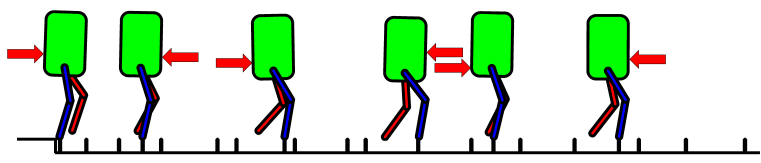

(e) Changing Step Length with Perturbation

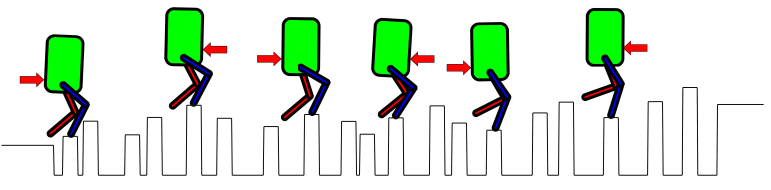

(f) Changing both Step Length an Step Height with Perturbation

Fig. 2: The problem of dynamically walking over a randomly generated set of discrete footholds. Simulation video: https://youtu.be/Pxhb4_ojiC8.

linear hybrid dynamic model of the robot that can achieve precise foot placement with single-step changes on step length and step height. We firstly pre-compute a gait library that is populated with a small number of periodic gaits with respect to different values of initial and resulting step length and step height, while satisfying torque limits, ground reaction forces and other key constraints. Instead of precomputing transition controllers between discrete elements of the gait library, at the beginning of a step, the desired footstep placement of the next stepping stone is determined, and based on this, the gait library is linearly interpolated to provide a desired gait based on the step length and step height of the next step as well as those of the current robot's configuration. This work builds off recent work on periodic walking gait libraries in [5], [6]. In comparison to prior work, this paper makes the following additional contributions:

- We present 2-step periodic gait optimization and a gaitlibrary-interpolation approach for achieving a contin- uum of desired step lengths and step heights.

- 2-step periodic gait optimization takes into account not only the footstep placement of the next step but also current configuration of the robot, allowing us to handle the step transition when switching between different walking gaits.

- The gait interpolation policy requires a small number of gaits (4 gaits for the problem of randomly-varying either step length or step height, and 16 gaits for the problem of randomly-varying both step length and step height).

- Numerical validations on different terrains (see Fig. 2):

(a) Randomly-varying step length only within the range of [20:90] $(\mathrm{cm})$ with the precision of only 2 (cm).

(b) Randomly-varying step height only within the range of [-30:30] $(\mathrm{cm})$ where the step length is constant at $50(\mathrm{~cm})$.

(c) Randomly-varying both step length and step height at the same time where the range of step length and step height are [30:80] $(\mathrm{cm})$ and [-30:30] $(\mathrm{cm})$ respectively.

(d) Planar version of the W-Prize terrain [1].

$(e, f)$ Force perturbation added to the cases of $(a, c)$.

- Experimental validation on ATRIAS robot for the problem of changing step length within the range of [23 : 78] $\mathrm{cm}$.

We believe that this is the first work that successfully experimentally demonstrates the problem of dynamic walking on stepping stones for a bipedal or humanoid robot. Unlike prior works on quasi-static walking using ZMP (for example [12],[13]), our experiment handles a large change in step length (within the range of [23:78] $\mathrm{cm}$ ) and fast walking speed (average speed of $0.6 \mathrm{~m} / \mathrm{s}$ ).

The remainder of the paper is organized as follows. Section II presents the hybrid dynamical model of 2D ATRIAS, an underactuated planar bipedal robot. Section III presents background on periodic gait optimization using Hybrid Zero Dynamics and input-ouput linearizing controller. Section IV presents our proposed approach on 2-step periodic gait optimization and a gait library interpolation strategy. Section V]presents numerical validation of the controller on ATRIAS. Finally, Section VII provides concluding remarks.

\section{DyNAMiCAL MODEL FOR WALKING}

The bipedal robot shown in Fig. 3 is a planar representation of ATRIAS. Its total mass is $63 \mathrm{~kg}$, with approximately $50 \%$ of the mass in the hips and $40 \%$ in the torso, and with light legs formed by a four-bar linkage. The robot is approximately left-right symmetric.

The configuration variables for the system can be defined as $q:=\left(q_{T}, q_{1 R}, q_{2 R}, q_{1 L}, q_{2 L}\right) \in \mathbb{R}^{5}$. The variable $q_{T}$ corresponds to the world frame pitch angle, while the variables $\left(q_{1 R}, q_{2 R}, q_{1 L}, q_{2 L}\right)$ refer to the local coordinates for linkages. The subscripts $L$ and $R$ refers to left and right legs. Fig. 3 illustrates $q_{1}, q_{2}$ angles for one of the legs. Each 


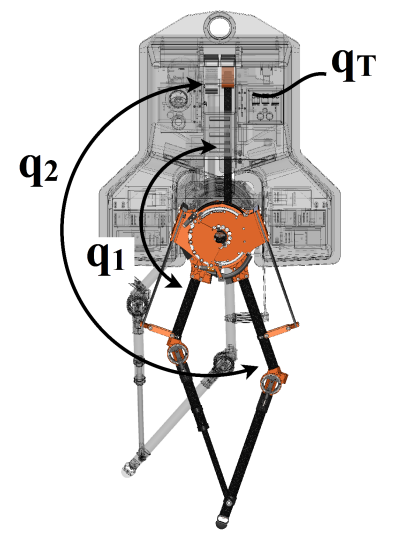

Fig. 3: Biped coordinates. The world frame pitch angle is denoted by $q_{T}$, while $\left(q_{1}, q_{2}\right)$ are body coordinates. The model is assumed left-right symmetric.

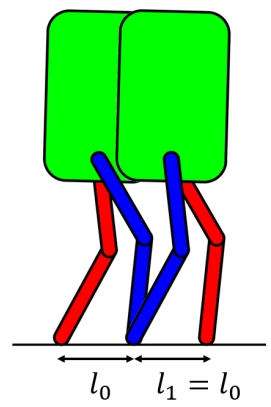

Fig. 4: Periodic walking gait has the resulting step length $\left(l_{1}\right)$ similar to the initial step length $\left(l_{0}\right)$, or in other words $l_{1}=l_{0}$.

of the four linkages are actuated by a DC motor behind a 50:1 gear ratio harmonic drive, with the robot having one degree of underactuation. The four-bar linkage mechanism comprising of the leg coordinates $\left(q_{1}, q_{2}\right)$ map to the leg angle and knee angle $\left(q_{L A}, q_{K A}\right)$, as $q_{L A}:=\frac{1}{2}\left(q_{1}+q_{2}\right)$ and $q_{K A}:=q_{2}-q_{1}$. The state $x$ denotes the generalized positions and velocities of the robot and $u$ denotes the joint torques. A hybrid model of walking can be expressed as

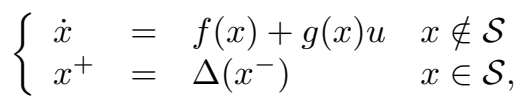

where $\mathcal{S}$ is the impact surface and $\Delta$ is the reset or impact map. A more complete description of the robot and a derivation of its model are given in [17].

\section{Periodic WALKING WITH HYbRID Zero DyNAMICS}

Having described the dynamical model of ATRIAS, we will now present the background on optimization for periodic gait design using virtual constraints and input-output linearization, a nonlinear feedback controller to exponentially stabilize the closed-loop system.

\section{A. Periodic Gait Design Using Virtual Constraints}

The nominal feedback controller is based on the virtual constraints framework presented in [19]. Virtual constraints are kinematic relations that synchronize the evolution of
TABLE I: Optimization constraints

$\begin{array}{cl}\text { Motor Toque } & |u| \leq 7 \mathrm{Nm} \\ \text { Impact Impulse } & F_{e} \leq 15 \mathrm{Ns} \\ \text { Friction Cone } & \mu \leq 0.6 \\ \text { Vertical Ground Reaction Force } & F_{s t}^{v} \geq 200 \mathrm{~N} \\ \text { Mid-step Swing Foot Clearance } & \left.h_{f}\right|_{s=0.5} \geq 0.1 \mathrm{~m}\end{array}$

the robot's coordinates via continuous-time feedback control. One virtual constraint in the form of a parametrized spline can be imposed for each (independent) actuator. Parameter optimization is used to find the spline coefficients so as to create a periodic orbit satisfying a desired step length, while respecting physical constraints on torque, motor velocity, and friction cone. Since the gait is periodic, the initial step length and the resulting step length must be the same (see Fig. 4 . The optimizer used here is based on the direct collocation framework from [11], although other optimization methods, such as [10] or fmincon can be used as well.

The virtual constraints are expressed as an output vector

$$
y=h_{0}(q)-h_{d}(s(q), \alpha),
$$

to be asymptotically zeroed by a feedback controller. Here, $h_{0}(q)$ specifies the quantities to be controlled

$$
h_{0}(q)=\left[\begin{array}{c}
q_{L A}^{s t} \\
q_{K A}^{s t} \\
q_{L A}^{s w} \\
q_{K A}^{s w}
\end{array}\right],
$$

where st and sw designate the stance and swing legs, respectively, and $h_{d}(s, \alpha)$ is a 4-vector of Beziér polynomials in the parameters $\alpha$ specifying the desired evolution of the $h_{0}(q)$, where $s$ is a gait phasing variable defined as

$$
s:=\frac{\theta-\theta_{\text {init }}}{\theta_{\text {final }}-\theta_{\text {init }}},
$$

with $\theta=q_{T}+q_{L A}^{s t}$ being the absolute stance leg angle.

The cost function and constraints for the optimization are formulated as in [19] [Chap. 6.6.2], with the constraints given in Table $[$ and the cost taken as the integral of squared torques over step length:

$$
J=\int_{0}^{T}\|u(t)\|_{2}^{2} d t
$$

In addition to the above constraints, we also need to guarantee the periodicity of the gait:

- The initial state at start of the first step is given by $x=x_{0}^{+}$with corresponding (initial) step length of $l_{0}$.

- The state at end of the first step (before impact) is given by $x=x_{1}^{-}$with corresponding (resulting) step length of $l_{1}$.

- Impact constraints at the end of the step are enforced as $x_{1}^{+}=\Delta\left(x_{1}^{-}\right)$.

- Periodic constraints are then enforced as $x_{1}^{+}=x_{0}^{+}$, resulting in $l_{1}=l_{0}$.

Here, the superscript '-' and ' + ' represent the state right before and right after the impact, and $\Delta$ is the reset or impact map from 1 . 


\section{B. Input-output linearization}

The optimization results in a desired walking gait encoded through $h_{d}(s(q), \alpha)$ in (2) and therefore our control goal is to drive $y(q) \rightarrow 0$. In our method, we use input-output linearization, a nonlinear feedback controller to enforce exponential stability for the system [2]. If $y(q)$ has vector relative degree 2 , then the second derivative takes the form

$$
\ddot{y}=L_{f}^{2} y(q, \dot{q})+L_{g} L_{f} y(q, \dot{q}) u .
$$

We can then apply the following pre-control law

$$
u(q, \dot{q})=u^{*}(q, \dot{q})+\left(L_{g} L_{f} y(q, \dot{q})\right)^{-1} \mu,
$$

where

$$
u^{*}(q, \dot{q}):=-\left(L_{g} L_{f} y(q, \dot{q})\right)^{-1} L_{f}^{2} y(q, \dot{q}),
$$

and $\mu$ is a stabilizing control to be chosen. Defining transverse variables $\eta=[y, \dot{y}]^{T}$, and using the IO linearization controller above with the pre-control law (7), we have,

$$
\ddot{y}=\mu \text {. }
$$

The exponential convergence of the control output $y$ then can be easily derived using PD controller:

$$
\mu=-K_{p} y-K_{d} \dot{y} .
$$

Having presented the background on periodic gait optimization using Hybrid Zero Dynamics and input-output linearization, we now introduce our proposed approach using 2-step periodic gait optimization to handle randomly-varying discrete terrain resulting in consecutive changes in step length and step height at each walking step.

\section{2-Step PERIOdic GAIT Design USING ViRTUAL CONSTRAINTS}

Inspired by the main issue of step transition on stepping stones [16], we develop an optimization framework to design 2-step periodic walking gaits, taking into account not only the desired footstep location of the next step but also the current configuration of the robot. The method combines virtual constraints, parameter optimization, and an interpolation strategy for creating a continuum of gaits from a finite library of gaits. The notion of a 2-step periodic gait means that the robot states are designed to be converge back to the initial condition after 2 walking steps. To be more specific, we will start off with the problem of changing step length only or walking on flat ground with varied step length.

1) Changing Only Step Lengths: In the nominal problem of periodic optimization presented in Section III-A, we need to optimize for only one walking step with the constraint on the resulting step length $\left(l_{1}\right)$ to be equal to the initial step length $\left(l_{0}\right)$ (see Fig. 4). For this problem, we use the same optimization framework discussed in III-A but we will optimize for 2 walking steps while following additional constraints that allows us to have different step lengths during transition (see Fig. 5):

- The initial state at start of the first step is given by $x=x_{0}^{+}$with corresponding (initial) step length $l_{0}$.

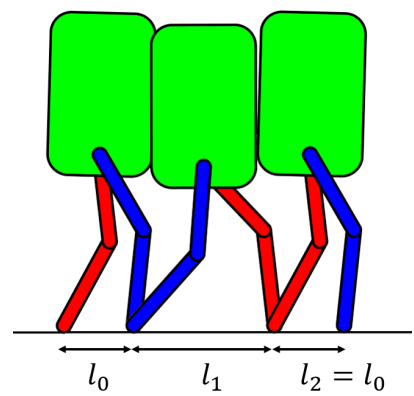

Fig. 5: 2-Step periodic walking with changing step lengths only. The walking gait is 2-step periodic therefore the step length of the second step and that of the initial condition are the same $\left(l_{2}=l_{0}\right)$.

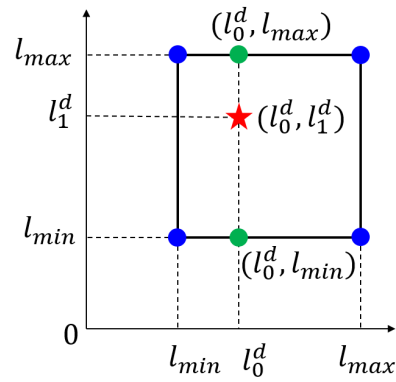

Fig. 6: Gait interpolation for the problem of changing step length only.

- The state at the end of the first step (before impact) is $x=x_{1}^{-}$with (resulting) step length $l_{1}$.

- Impact constraints at the end of the first step are enforced as $x_{1}^{+}=\Delta\left(x_{1}^{-}\right)$.

- The initial state at start of the second step is given by $x=x_{1}^{+}$with corresponding (initial) step length of $l_{1}$.

- The state at the end of the second step (before impact) is $x=x_{2}^{-}$with (resulting) step length of $l_{2}$.

- Impact constraints at the end of the second step are enforced as $x_{2}^{+}=\Delta\left(x_{2}^{-}\right)$.

- Periodic constraints are then enforced as $x_{2}^{+}=x_{0}^{+}$, resulting in $l_{2}=l_{0}$.

The optimization problem is then used to generate a gait library with different values of $l_{0}$ and $l_{1}$. In this work, we optimize four different gaits corresponding to:

$$
\left\{\begin{array}{l}
\left(l_{0}, l_{1}\right)=(0.3,0.3) m \\
\left(l_{0}, l_{1}\right)=(0.3,0.7) m \\
\left(l_{0}, l_{1}\right)=(0.7,0.3) m \\
\left(l_{0}, l_{1}\right)=(0.7,0.7) m .
\end{array}\right.
$$

This is similar to precomputing four gait primitives corresponding to walking with small steps $\left(\left(l_{0}, l_{1}\right)=\right.$ $(0.3,0.3) \mathrm{m})$, switching from a small step to a large step $\left(\left(l_{0}, l_{1}\right)=(0.3,0.7) \mathrm{m}\right)$, switching from a large step to a small step $\left(\left(l_{1}, l_{0}\right)=(0.7,0.3) \mathrm{m}\right)$ and walking with large steps $\left(\left(l_{0}, l_{1}\right)=(0.7,0.7) m\right)$. Having a gait library with different gaits representing a few general motion primitives, we then do gait interpolation to get the desired walking gait with an arbitrary set of $\left(l_{0}^{d}, l_{1}^{d}\right)$. 


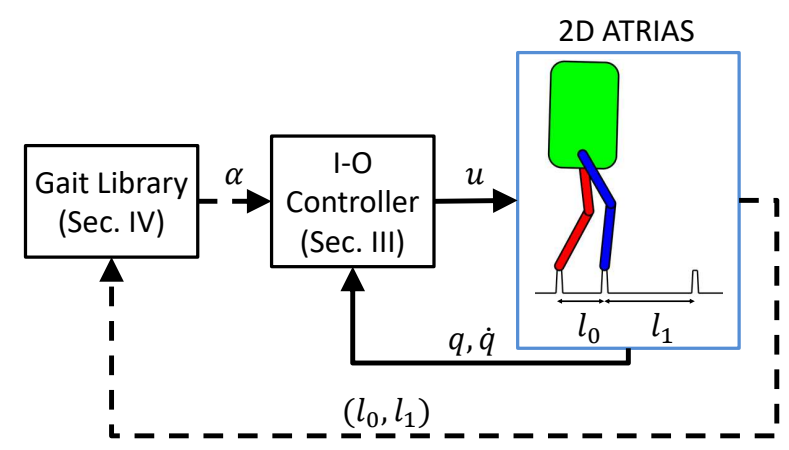

Fig. 7: Diagram of the controller structure for the problem of changing step length only, integrating the gait library and I-O linearization controller. Solid lines represent signals in continuous time; dashed lines represent signals in discrete time.

Let $\alpha\left(l_{0}^{d}, l_{1}^{d}\right)$ be the Beziér coefficients (defined in (2)) of the desired walking gait that has the initial step length $l_{0}^{d}$ and the resulting step length $l_{1}^{d}$. If $l_{0}^{d} \in[0.3: 0.7] \mathrm{m}$ and $l_{1}^{d} \in[0.3: 0.7] m$, we will compute $\alpha\left(l_{0}^{d}, l_{1}^{d}\right)$ using bilinear interpolation of the coefficients from the four nominal gait parameters precomputed using optimization. Therefore the new interpolated gait can be derived in real-time $(1 \mathrm{kHz}$ in our experiment) right after the impact of each walking step. A detailed explanation for bilinear interpolation can be found in [21], and summarized in Fig. 6 In this work, we use the MATLAB function "interp2" to implement the algorithm.

Remark 1: If $l_{0} \notin[0.3: 0.7] m$ or $l_{1} \notin[0.3: 0.7] m$, we can use extrapolation to compute the gait parameters for the desired gait.

The gait library and gait interpolation are used to update the walking gait for every walking step based on the desired footstep placement of the next step $\left(l_{1}\right)$ and the current configuration of the robot $\left(l_{0}\right)$. They are then incorporated using input-output linearization to control the robot to follow the updated walking gait. The closed-loop control diagram is shown in Fig. 7

Remark 2: Note that the proposed method has a resemblance to MPC. While we use a 2-step periodic gait, we switch the gait at the end of each step, i.e., half-way into the 2 -step periodic gait. For instance, with current step length being $l_{0}$, and subsequent step lengths being $l_{1}, l_{2}$, we use a gait with $\left(l_{0}, l_{1}\right)$ and switch at the end of the first step to $\left(l_{1}, l_{2}\right)$ so that there is an overlap of one step between the gaits. This easily addresses gait transitions that typically cause large violations in unilateral force constraints, friction constraints, and torque constraints.

Remark 3: Also note that the authors of [16] use control barrier functions to handle gait transitions. While this appears to work well, the feasibility of the quadratic program that enforces the control barrier constraint is not guaranteed. In this present work, as we will see, we achieve better results without using control barrier functions. We can easily add control barrier functions on top of the current method to further enforce these safety-critical constraints. Since the underlying method achieves the foot placement without

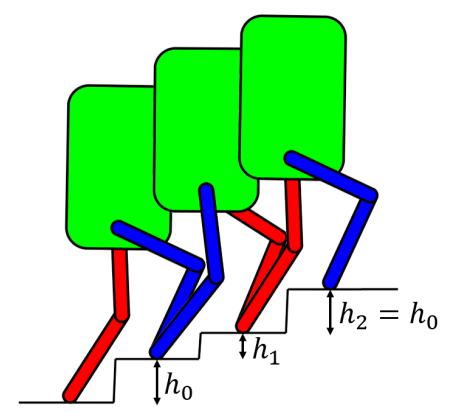

Fig. 8: 2-Step periodic walking with changing step heights only. The walking gait is 2-step periodic therefore the step height of the second step and that of the initial condition are the same $\left(h_{2}=h_{0}\right)$. Note that step heights $h_{0}, h_{1}$ can be positive (stepping up) or negative (stepping down). In this figure, we illustrate the case of both $h_{0}$ and $h_{1}$ being positive for convenience.

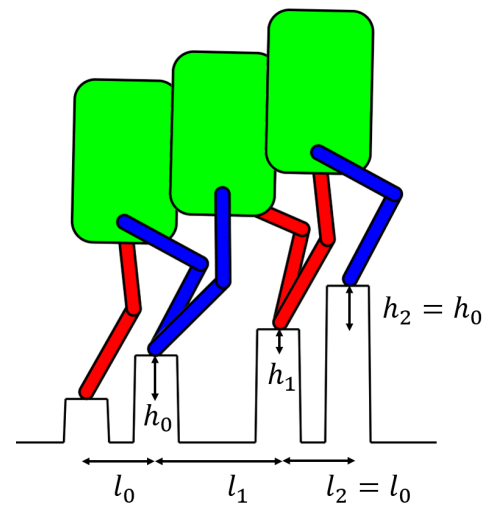

Fig. 9: 2-Step periodic walking with changing step lengths and step heights. The walking gait is 2-step periodic therefore the step length and step height of the second step and that of the initial condition are the same $\left(l_{2}=l_{0}, h_{2}=h_{0}\right)$. Note that step heights $h_{0}, h_{1}$ can be positive (stepping up) or negative (stepping down). In this figure, we illustrate the case of both $h_{0}$ and $h_{1}$ being positive for convenience.

requiring the barriers, the barriers will remain inactive most of the time, leading to better feasibility of the quadratic program.

2) Changing Only Step Heights: For the problem of changing step heights only, the framework presented for changing step length on flat ground in Section IV-.1 can be applied where $\left(h_{0}, h_{1}\right)$ plays the role of $\left(l_{0}, l_{1}\right)$ (see Fig. 8). For the problem of changing step length on flat ground, the step height is zero. In the problem of changing step height only in Fig. 8, we assume that the step length or the width of the stair is constant.

3) Changing Both Step Lengths and Step Heights: We now can combine the methods presented in Section IV-.1 and IV-.2 to handle the problem of walking on stepping stones with varied step length and step height for every walking step (see Fig. 9). Since the gait parameters now depend on 4 variables $l_{0}, h_{0}, l_{1}, h_{1}$, the gait interpolation needs to be extended for 4 variables and the number of gaits increases to $2^{4}=16$ gaits. 
Remark 4: Note that each of the nominal periodic walking gaits presented in Section III is locally exponentially stable [20]. The stability condition for switching policies between different locally exponentially stable periodic gaits can be found in [15], wherein it is assumed that one periodic gait switches into the domain of attraction of a subsequent periodic gait. With the 2-step periodic gaits, we can guarantee that when we switch to the next gait, the initial state of the robot is close to the periodic orbit of the next gait.

Remark 5: Creating an interpolated gait between two nearby periodic gaits ensures that constraints enforced by the periodic gaits are enforced by the interpolated gait as well due to local continuity of the constraints. Further work needs to be carried out to establish stability results for switching between interpolated gaits.

\section{NumeriCAl VALIDATION}

In this Section, we will demonstrate the effectiveness of the proposed method by conducting numerical simulations on the model of ATRIAS.

Using our method, we can control our robot to overcome different type of terrains:

- Changing step length only within the range of [20:90] $(\mathrm{cm})$ with the precision of only $2(\mathrm{~cm})$ (see Fig. 2a)

- Changing step height only within the range of [-30:30] $(\mathrm{cm})$ where the step length is constant at $50(\mathrm{~cm})($ see Fig. 2b

- Changing step length and step height at the same time where the range of step length and step height are [30:80] $(\mathrm{cm})$ and [-30:30] $(\mathrm{cm})$ respectively (see Fig. 2c)

- Planar version of the W-Prize terrain [1] (see Fig. 2c)

- Changing step length with perturbation (see Fig. 2e, where the perturbation is generated by applying horizontal external force of $\pm 300 \mathrm{~N}$ on the robot torso with the duration of $0.2 s$ in the middle of some steps.

- Changing step length and step height with perturbation (see Fig. 2f), with similar type of perturbation mentioned above but the magnitude of the external force is now $200 N$.

In all simulations, we check constraints on footstep placement, friction constraints and input saturation stated in Table 1. Note that friction constraints are checked for both impulse at impact and contact force during the continuous phase. Fig. 11 shows the satisfactions of those constraints in one example of ATRIAS walking on randomly generated discrete footholds shown in Fig. 2c, where step length and step height are varied in the range of [30:80] $(\mathrm{cm})$ and [-30:30] $(\mathrm{cm})$ respectively. In this simulation the absolute error between the desired step length and the real step length has the minimum of $0 \mathrm{~m}$, the maximum of $0.0453 \mathrm{~m}$ and the mean of $0.0113 \mathrm{~m}$.

With the problem of changing step length only, we also compare the performance of (a) our prior work on Control Barrier Functions and gait library presented in [16] and (b) our proposed method of the 2-step periodic gait library. Both controllers are run on the same terrain illustrated in Fig. $2 \mathrm{a}$.
From Fig. 12, we can clearly see that our proposed method of 2-step periodic gait library (thick red line) has vertical ground reaction force $\left(F_{s t}^{v}\right)$ with smaller peak amplitudes, coefficient $k=\left|F_{v t}^{h} / F_{v t}^{v}\right|$ that stays more inside the friction cone, and control inputs with smaller norm. Note that although these two controllers are applied on the same terrain, the walking step times are different because the gait libraries and the low-level controllers are different.

In Fig. 10, we demonstrate the robot walking over multiple terrains including:

(1) Worst case of walking up and down with large step length,

(2) Worst case of walking up and down with small step length,

(3) 20 steps walking over randomly generated terrain with stone size of $25(\mathrm{~cm})$,

(4) 20 steps walking over randomly generated terrain with stone size of $5(\mathrm{~cm})$.

Note that we use the same controller with the same gait library for all these different terrains, thereby establishing that our single controller can handle different types of variation in the terrain, including step length, step height, as well as stone size. In particular, with Terrain (4), the random terrain with stone size of $5 \mathrm{~cm}$, we show the accuracy of the precise footstep placements. With Terrain (3), the random terrain with stone size of $25 \mathrm{~cm}$, we show that the robot has a good swing foot clearance to avoid the corners of the larger stepping stones.

Here, we also sucessfully applied our proposed control method for the planar version of the W-Prize terrain listed in [1], which is made from placing cinder blocks with distances varied in [35: 98] $(\mathrm{cm})$. There are also stepping-up and stepping-down stones at the start and the end of the terrain (see Fig. 2d). Note that, in the simulation, all the physical constraints are checked except the constraint on avoiding the cinder blocks from tipping over. This additional challenge of the blocks tipping over is not addressed in this paper but it is an interesting problem to consider in future work.

\section{EXPERIMENTAL VALIDATION}

In this Section, we will present experimental results on ATRIAS walking over stepping stones (see Fig 1). The circle is covered by 24 stepping stones with the following distances or desired step lengths of the robot:

$$
\begin{aligned}
L_{d}= & {[56,31,64,78,33,75,30,40,72,67,35,23,} \\
& 33,52,76,50,42,78,37,31,51,76,74,69](\mathrm{cm}) .
\end{aligned}
$$

The controller is allowed knowledge of this information only for one step ahead.

For this paper, we present the experiment on changing step length only. Future works will consider experiments on dynamic walking while changing both step length and step height.

Fig. 13 shows the experiment data including step length (the horizontal distance between swing and stance foot), the 


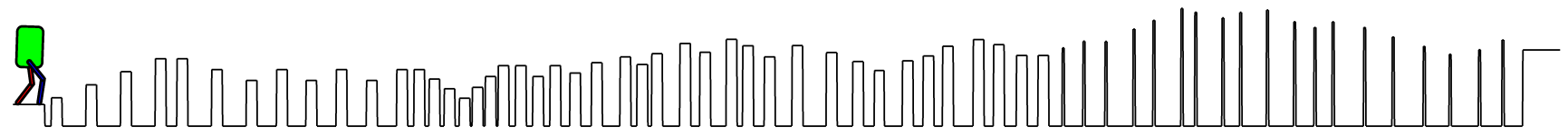

(1)

(2)

(3)

(4)

Fig. 10: ATRIAS walking on different terrains. (1) worst case of walking up and down with large step length. (2) worst case of walking up and down with small step length. (3) 20 walking steps over randomly generated terrain with stone size of $25(\mathrm{~cm})$ and then. (4) 20 walking steps over randomly generated terrain with stone size of $5(\mathrm{~cm})$. Simulation video: https://youtu.be/Pxhb4_ojic8.

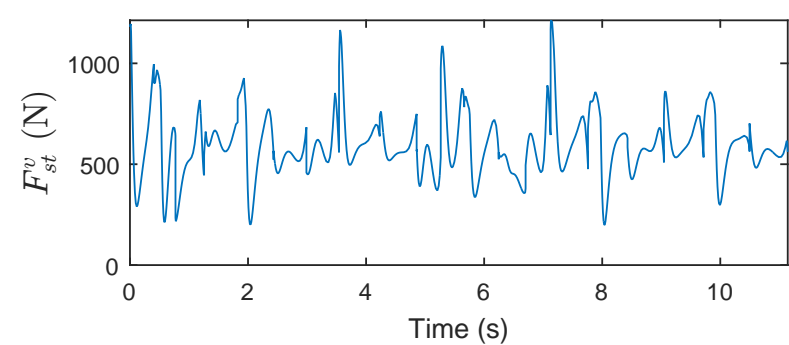

(a) Ground Reaction Force

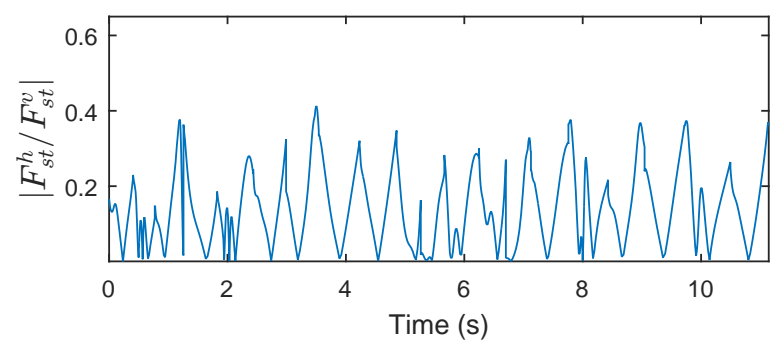

(b) Friction Cone

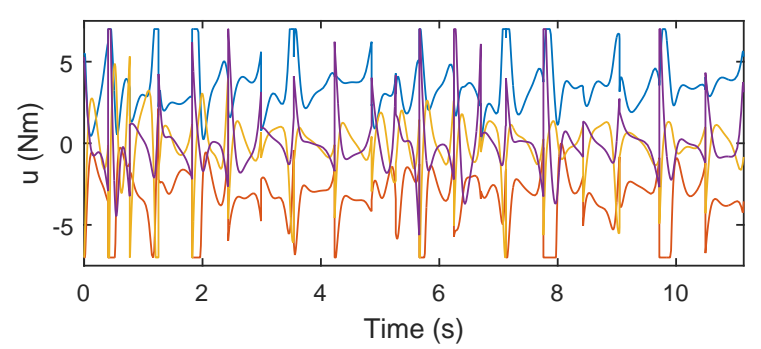

(c) Control Inputs

Fig. 11: Simulation of ATRIAS walking on randomly generated stepping stones with step length and step height changing in the range of [30:80] $(\mathrm{cm})$ and $[-30: 30](\mathrm{cm})$ respectively. The terrain is illustrated in Fig. $2 \mathrm{c}$. The following constraints are enforced: (a) Ground reaction force: $F_{s t}^{v} \geq 150(N)$; (b) Friction cone: $\left|F_{s t}^{h} / F_{s t}^{v}\right| \leq 0.6$; and (c) Control motor inputs saturated at $7(\mathrm{Nm})(|u| \leq 7)$. Note that there is a 50:1 gear ratio from the motors to the links.

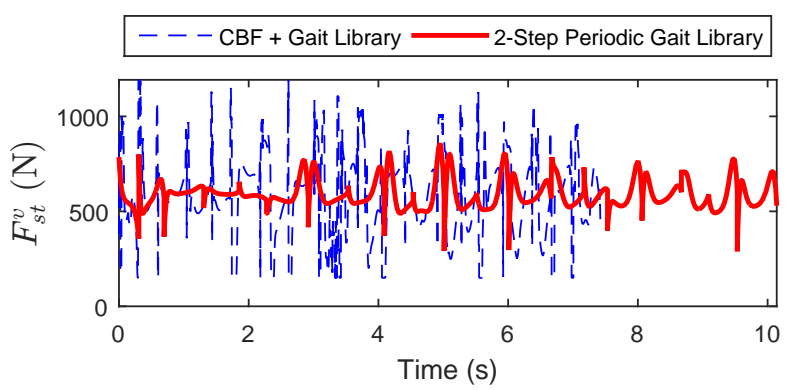

(a) Ground Reaction Force

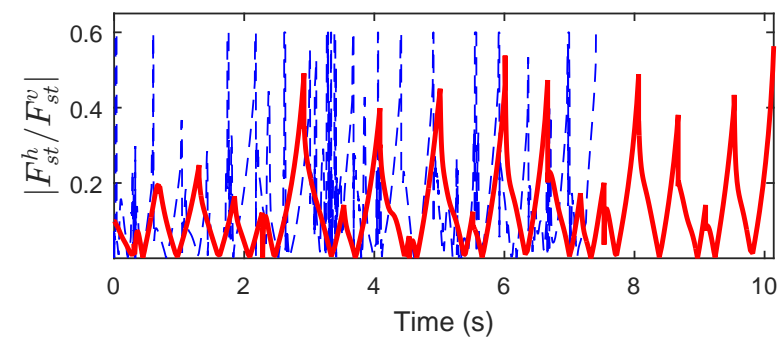

(b) Friction Cone

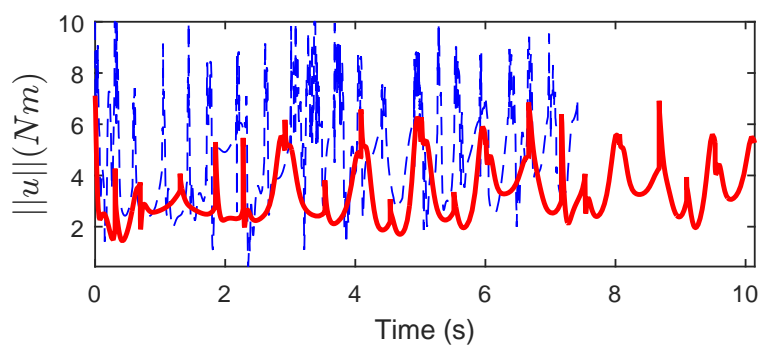

(c) Norm of Control Inputs

Fig. 12: We compare the "CBF + Gait Library" controller from [16] with the proposed "2-Step Periodic Gait Library" controller (thick red line). As is seen, the proposed controller has better ground reaction force with smaller peak amplitudes, lower friction requirements, as well as smaller control inputs. The comparison is made by simulating both controllers for the same terrain illustrated in Fig. 2a. 


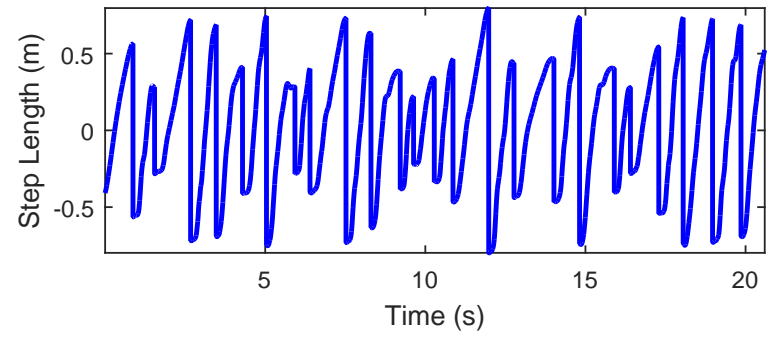

(a) Step Length

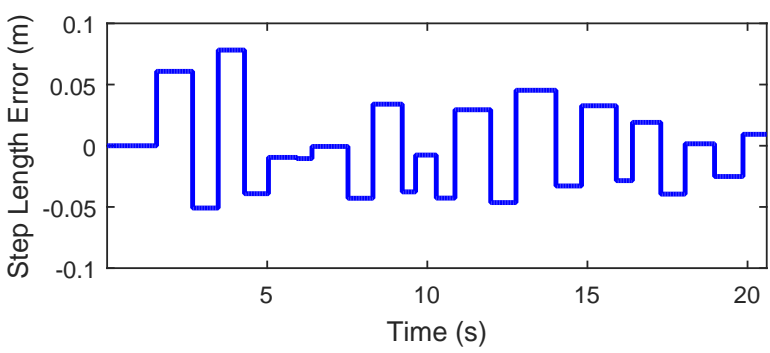

(b) Distance of the Foot from the Center of the Stone

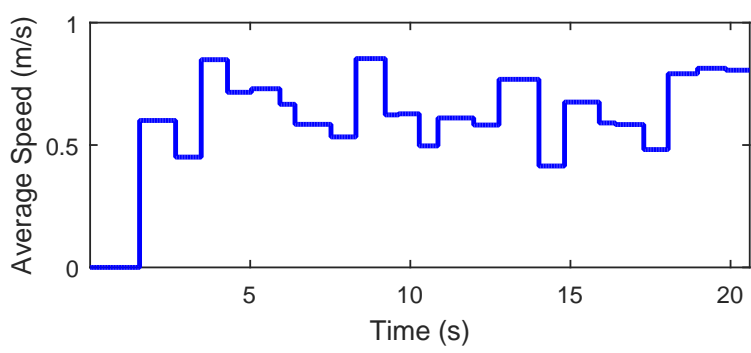

(c) Distance of the Foot from the Center of the Stone

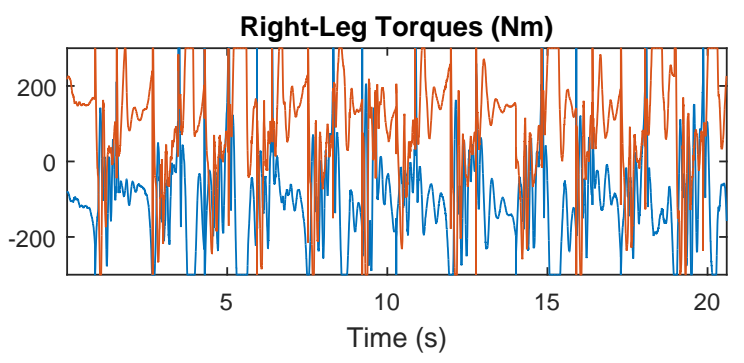

(d) Right-Leg Torques

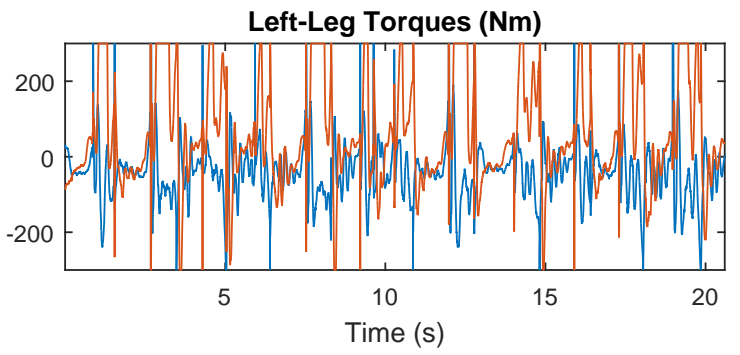

(e) Left-Leg Torques

Fig. 13: Results from experiment of ATRIAS walking over stepping stones. Experimental video: https://youtu.be/JKkPWHm6H7k step length error (the error between the stance foot position and the center of the stone), the average speed of walking for each step and the joint torques of the right and left leg respectively. The step length errors are always within the stone size of $[-10: 10](\mathrm{cm})$. The experiment was conducted with input saturation of $300(\mathrm{Nm})$ on joint torques. The average walking speed of the total 24 steps is $0.6(\mathrm{~m} / \mathrm{s})$. This experiment thus validated the effectiveness of our proposed method for the problem of dynamic walking on stepping stones with a wide range of step length (within [23:78] $\mathrm{cm}$ ) and a fast average walking speed $(0.6 \mathrm{~m} / \mathrm{s})$.

In the experiment, we estimate the stance foot position using the joint encoders and the IMU attached to the robot. To be more specific, the stance foot position with respect to the initial hip position is computed as follows:

$$
p_{\text {stancefoot }}=q_{\text {yaw }}^{\text {robot }} \times l_{\text {boom }}+d_{\text {hip } \rightarrow \text { stancefoot }},
$$

where $q_{\text {yaw }}^{\text {robot }}$ is the yaw angle of the robot measured from the IMU signal, $l_{\text {boom }}=2 \mathrm{~m}$ is the boom length and $d_{\text {hip } \rightarrow \text { stancefoot }}$ is the distance from the hip to stance foot is computed based on the joint encoders. Note that we do not make use of any external sensors (such as a boom encoder.)

\section{CONCLUSION}

We have presented a novel approach called 2-step periodic gait optimization that allows us to handle a wide change in footstep placement of the robot (both step length and step height). Since our walking gait optimization takes into account not only the upcoming terrain but also the current configuration of the robot, the method can effectively address the transition when the controller switches between different gaits. The gait library is pre-computed with a small number of gaits (4 gaits for the problem of changing step length or step height only and 16 gaits for the problem of changing both), then gait interpolation is implemented in real-time to adapt with random changes in the terrain as well as the initial condition of the robot. We successfully validated the proposed approach on the model of ATRIAS, an underactuated bipedal robot, under different types of terrain, including changing step length in the range of [20:90] $(\mathrm{cm})$ with the precision of only $2(\mathrm{~cm})$; changing step height only in the range of [-30:30] $(\mathrm{cm})$ or changing both step length and step height in the range of [30:80] $(\mathrm{cm})$ and [-30:30] $(\mathrm{cm})$ respectively. A 2D version of the W-Prize terrain was also attempted. In future work, this method will be extended to $3 \mathrm{D}$ walking.

\section{REFERENCES}

[1] "The W-Prize on stepping stones," http://www.wprize.org/SteppingStones.html.

[2] A. D. Ames, K. Galloway, K. Sreenath, and J. W. Grizzle, "Rapidly exponentially stabilizing control lyapunov functions and hybrid zero dynamics," IEEE Transactions on Automatic Control (TAC), vol. 59, no. 4, pp. 876-891, April 2014.

[3] J. Chestnutt, J. Kuffner, K. Nishiwaki, and S. Kagami, "Planning biped navigation strategies in complex environments," in IEEE International Conference on Humanoid Robotics, 2003, pp. 117-123. 
[4] J. Chestnutt, M. Lau, G. Cheung, J. Kuffner, J. Hodgins, and T. Kanade, "Footstep planning for the honda asimo humanoid," Proceedings of the 2005 IEEE International Conference on Robotics and Automation., pp. 629 - 634, 2005.

[5] X. Da, O. Harib, R. Hartley, B. Griffin, and J. Grizzle, "From 2d design of underactuated bipedal gaits to $3 \mathrm{~d}$ implementation: Walking with speed tracking," IEEE Access, vol. PP, no. 99, pp. 1-1, 2016.

[6] X. Da, R. Hartley, and J. W. Grizzle, "First steps toward supervised learning for underactuated bipedal robot locomotion, with outdoor experiments on the wave field," in IEEE International Conference on Robotics and Automation (ICRA), 2017.

[7] R. Deits and R. Tedrake, "Footstep planning on uneven terrain with mixed-integer convex optimization." Proceedings of the 2014 IEEE/RAS International Conference on Humanoid Robots, pp. 279286, 2014

[8] R. L. H. Deits, "Convex segmentation and mixed-integer footstep planning for a walking robot," Master's thesis, Massachusetts Institute of Technology, 2014.

[9] R. Desai and H. Geyer, "Robust swing leg placement under large disturbances," Proceedings of the IEEE International Conference on Robotics and Biomimetics (ROBIO), pp. 265-270, 2012.

[10] A. Hereid, C. M. Hubicki, E. A. Cousineau, J. W. Hurst, and A. D. Ames, "Hybrid zero dynamics based multiple shooting optimization with applications to robotic walking," in 2015 IEEE International Conference on Robotics and Automation (ICRA). IEEE, 2015, pp. 5734-5740.

[11] M. S. Jones, "Optimal control of an underactuated bipedal robot," Master's thesis, Oregon State University, ScholarsArchive@OSU, 2014.

[12] S. Kajita, F. Kanehiro, K. Kaneko, K. Fujiwara, K. Harada, K. Yokoi, and H. Hirukawa, "Biped walking pattern generation by using preview control of zero-moment point," Proceedings of the IEEE International Conference on Robotics and Automation (ICRA), vol. 2, pp. 1620 1626, 2003.
[13] J. J. Kuffner, K. Nishiwaki, S. Kagami, M. Inaba, and H. Inoue, "Footstep planning among obstacles for biped robots," Proceedings of the 2001 IEEE/RSJ International Conference on Intelligent Robots and Systems, , vol. 1, pp. 500 - 505, 2001.

[14] P. Michel, J. Chestnutt, J. Kuffner, and T. Kanade, "Vision-guided humanoid footstep planning for dynamic environments," in Humanoids, 2005, pp. 13-18

[15] M. S. Motahar, S. Veer, and I. Poulakakis, "Composing limit cycles for motion planning of $3 \mathrm{~d}$ bipedal walkers," in the IEEE Conference On Decision and Control (CDC), 2016.

[16] Q. Nguyen, X. Da, J. W. Grizzle, and K. Sreenath, "Dynamic walking on stepping stones with gait library and control barrier," Workshop on Algorithimic Foundations of Robotics, 2016.

[17] A. Ramezani, J. W. Hurst, K. Akbari Hamed, and J. W. Grizzle, "Performance Analysis and Feedback Control of ATRIAS, A ThreeDimensional Bipedal Robot," Journal of Dynamic Systems, Measurement, and Control, vol. 136, no. 2, 2014.

[18] M. Rutschmann, B. Satzinger, M. Byl, and K. Byl, "Nonlinear model predictive control for rough-terrain robot hopping," Proceedings of the IEEE/RSJ International Conference on Intelligent Robots and Systems (IROS), pp. 1859-1864, 2012.

[19] E. R. Westervelt, J. W. Grizzle, C. Chevallereau, J. Choi, and B. Morris, Feedback Control of Dynamic Bipedal Robot Locomotion, ser Control and Automation. Boca Raton, FL: CRC, June 2007.

[20] E. R. Westervelt, J. W. Grizzle, C. Chevallereau, J. H. Choi, and B. Morris, Feedback control of dynamic bipedal robot locomotion. CRC press Boca Raton, 2007.

[21] Wikipedia. [Online]. Available: https://en.wikipedia.org/wiki/Bilinear_ interpolation

[22] T. Yang, E. Westervelt, A. Serrani, and J. P. Schmiedeler, "A framework for the control of stable aperiodic walking in underactuated planar bipeds," Autonomous Robots, vol. 27, no. 3, pp. 277-290, 2009. 